\title{
A new formula for the coefficients of Gaussian polynomials
}

\author{
Dorin Andrica and Ovidiu Bagdasar
}

\begin{abstract}
We deduce exact integral formulae for the coefficients of Gaussian, multinomial and Catalan polynomials. The method used by the authors in the papers $[2,3,4]$ to prove some new results concerning cyclotomic and polygonal polynomials, as well as some of their extensions is applied.
\end{abstract}

\section{Introduction}

Let $n$ be a positive integer. In [4] we have studied the polygonal polynomials

$$
P_{n}(z)=(z-1)\left(z^{2}-1\right) \cdots\left(z^{n}-1\right), \quad n=1,2, \ldots,
$$

having integer coefficients and degree $\frac{n(n+1)}{2}$. Clearly, for each $k=1, \ldots, n$, the roots of $z^{k}-1$ are the complex coordinates of the vertices of the regular $k$-gon centered in the origin and having 1 as a vertex. Consequently, the roots of $P_{n}(z)$ are the complex coordinates of the vertices, with repetitions, of the regular $k$-gons, for $k=1, \ldots, n$.

For positive integers $m$ and $r$, the Gaussian polynomial is defined by

$$
\left(\begin{array}{c}
m \\
r
\end{array}\right)_{z}=\frac{P_{m}(z)}{P_{r}(z) P_{m-r}(z)}= \begin{cases}\frac{\left(z^{m-r+1}-1\right) \cdots\left(z^{m}-1\right)}{(z-1) \cdots\left(z^{r}-1\right)} & r \leq m \\
0 & r>m .\end{cases}
$$

Key Words: Gaussian polynomial, multinomial polynomial, Catalan polynomial, polygonal polynomial, integral formula.

2010 Mathematics Subject Classification: Primary 11B83; Secondary 26 C05.

Received: 02.01.2019

Accepted: 31.01 .2019 
While formula (2) may seem to involve a rational function, the division actually is exact in the ring $\mathbb{Z}[z]$, and it generates a polynomial of degree $r(m-r)$. There are numerous identities involving Gaussian polynomials, many of which are similar to those concerning the classical binomial coefficients.

The factorization of (2) in terms of cyclotomic polynomials was given by Knuth and Wilf [9], while Chen and Hou [7] showed that all the roots of the Gaussian polynomial are distinct. The factorization is

$$
\left(\begin{array}{c}
m \\
r
\end{array}\right)_{z}=\prod_{k=1}^{m}\left[\Phi_{k}(z)\right]^{\lfloor m / k\rfloor-\lfloor r / k\rfloor-\lfloor(m-r) / k\rfloor},
$$

where $\Phi_{k}(z)$ denotes the $k$ th cyclotomic polynomial.

Both the polygonal and Gaussian polynomials are special cases of a general class of polynomials introduced and studied in [3], namely

$$
F_{m_{1}, \ldots, m_{n}}^{z_{1}, \ldots, z_{n}}(z)=\prod_{k=1}^{n}\left(z^{m_{k}}-z_{k}\right),
$$

where $n \geq 2$ and $m_{1}, m_{2}, \ldots, m_{n}$ are positive integers, while $z_{1}, z_{2}, \ldots, z_{n}$ are complex numbers with $\left|z_{1}\right|=\left|z_{2}\right|=\cdots=\left|z_{n}\right|=1$.

Writing the Gaussian polynomial in algebraic form

$$
\left(\begin{array}{c}
m \\
r
\end{array}\right)_{z}=\sum_{j=0}^{r(m-r)} C_{j}^{(m, r)} z^{j}
$$

the coefficient $C_{j}^{(m, r)}$ has many combinatorial and algebraic interpretations. First, it represents the number of partitions of number $j$ whose Ferrers diagram fits into a $r \times(m-r)$ rectangle. Also, if $\delta=\delta_{1} \delta_{2} \cdots \delta_{r}$ is a $r$-subset of $[n]$, and $\sigma(\delta)=\sum_{i=1}^{r} \delta_{i}$ (weight of $\delta$ ), then the coefficient $C_{j}^{(m, r)}$ is the number of $r$-subsets of $[n]$ with weight $j+\frac{r(r+1)}{2}[10]$.

Also, it is known that the sequence $C_{j}^{(m, r)}$ is unimodal. This property has first been stated by Cayley in 1856 and proved by Sylvester in 1878. The first constructive proof was given in 1990 by O'Hara [10]. Some generalized Gaussian coefficients were also found to be unimodal in 1992 by Kirillov in [8].

Furthermore, Butler [6] established that $\left(\begin{array}{c}m \\ r\end{array}\right)_{z}$ is a log-concave sequence of polynomials, but Stanley [11] showed that $C_{j}^{(m, r)}, j=0, \ldots, r(m-r)$, is not always log-concave (see, e.g., $\left(\begin{array}{l}4 \\ 2\end{array}\right)_{z}=1+z+2 z^{2}+z^{3}+z^{4}$ ).

In this paper, we first derive an exact integral formula for the coefficients of the Gaussian polynomial, then obtain analogous formulae for the multinomial and the Catalan polynomial. These polynomials can also be recovered as particular instances of (4). Such integral formulae could be important for determining the asymptotic behaviour of the coefficients [12]. 


\section{A formula for the coefficients of Gaussian polynomials}

Let $z=\cos 2 t+i \sin 2 t$ for $t \in[0, \pi]$. For a positive integer $k$, using de Moivre's formula and Euler's exponential notation of complex numbers in polar form, we obtain:

$$
z^{k}-1=(\cos 2 k t-1)+i \sin 2 k t=2 i e^{i k t} \sin k t .
$$

Therefore, the Gaussian polynomial may be written as

$$
\begin{gathered}
\left(\begin{array}{c}
m \\
r
\end{array}\right)_{z}=\frac{(2 i)^{r} \prod_{k=1}^{r} e^{i(m-r+k) t} \sin (m-r+k) t}{(2 i)^{r} \prod_{k=1}^{r} e^{i k t} \sin k t} \\
e^{i r(m-r) t} \prod_{k=1}^{r} \frac{\sin (m-r+k) t}{\sin k t} .
\end{gathered}
$$

For what follows it is convenient to define the function

$$
\Lambda_{r}^{m}(t)=\prod_{k=1}^{r} \frac{\sin (m-r+k) t}{\sin k t}
$$

We now derive a new formula for the coefficients of the Gaussian polynomial.

Theorem 1. The coefficients of the polynomial $\left(\begin{array}{c}m \\ r\end{array}\right)_{z}$ are given by

$$
C_{j}^{(m, r)}=\frac{1}{\pi} \int_{0}^{\pi} \Lambda_{r}^{m}(t) \cdot \cos [r(m-r)-2 j] t \mathrm{~d} t, \quad j=0, \ldots, r(m-r) .
$$

Proof. Using formula (6) and the algebraic form of $\left(\begin{array}{c}m \\ r\end{array}\right)$, it follows that

$$
\left(\begin{array}{c}
m \\
r
\end{array}\right)_{z}=\sum_{k=0}^{r(m-r)} C_{k}^{(m, r)} z^{k}=\Lambda_{r}^{m}(t) \cdot e^{i r(m-r) t} .
$$

Using complex numbers in polar form, for $j=0 \ldots, r(m-r)$, we have

$$
C_{j}^{(m, r)}+\sum_{k \neq j} C_{k}^{(m, r)} z^{k-j}=z^{-j} \Lambda_{r}^{m}(t) \cdot e^{i r(m-r) t}=\Lambda_{r}^{m}(t) \cdot e^{i[r(m-r)-2 j] t},
$$

and integrating this relation over the interval $[0, \pi]$ we get the desired result.

In addition, the following relations are obtained

$$
\int_{0}^{\pi} \Lambda_{r}^{m}(t) \cdot \sin [r(m-r)-2 j] t \mathrm{~d} t=0, \quad j=0, \ldots, r(m-r) .
$$


Notice that the integral in formula (8) is not singular at $t=0$, since we have

$$
\lim _{t \rightarrow 0} \Lambda_{r}^{m}(t)=\prod_{k=1}^{r} \frac{m-r+k}{k}=\left(\begin{array}{c}
m \\
r
\end{array}\right) .
$$

Remark 2. By Theorem 1 one can easily show that the polynomial $\left(\begin{array}{c}m \\ r\end{array}\right)_{z}$ is palindromic. Indeed, for $j=0, \ldots, r(m-r)$, one obtains

$$
\begin{aligned}
C_{r(m-r)-j}^{(m, r)} & =\frac{1}{\pi} \int_{0}^{\pi} \Lambda_{r}^{m}(t) \cdot \cos [r(m-r)-2(r(m-r)-j)] t \mathrm{~d} t \\
& =\frac{1}{\pi} \int_{0}^{\pi} \Lambda_{r}^{m}(t) \cdot \cos [-r(m-r)+2 j] t \mathrm{~d} t=C_{j}^{(m, r)}
\end{aligned}
$$

One can also obtain elegant formulae for the middle coefficients.

Proposition 3. The middle coefficient of the polynomial $\left(\begin{array}{c}m \\ r\end{array}\right)_{z}$ is given by: 1. If $r(m-r)=2 k$, then

$$
C_{k}^{(m, r)}=\frac{1}{\pi} \int_{0}^{\pi} \Lambda_{k}^{m}(t) \mathrm{d} t
$$

2. If $r(m-r)=2 k+1$, then

$$
C_{k}^{(m, r)}=C_{k+1}^{(m, r)}=\frac{1}{\pi} \int_{0}^{\pi} \Lambda_{k}^{m}(t) \cdot \cos t \mathrm{~d} t
$$

A simple formula for the sum of these coefficients can also be obtained. The sum of coefficients for the Gaussian polynomial $\left(\begin{array}{c}m \\ r\end{array}\right)_{z}$ is

$$
\sum_{j=0}^{r(m-r)} C_{j}^{(m, r)}=\left(\begin{array}{c}
m \\
r
\end{array}\right)
$$

Indeed, for $k=1, \ldots, r$, we have the following limits

$$
\lim _{z \rightarrow 1} \frac{z^{m-r+k}-1}{z^{k}-1}=\frac{m-r+k}{k},
$$

and one obtains that

$$
\sum_{j=0}^{r(m-r)} C_{j}^{(m, r)}=\lim _{z \rightarrow 1}\left(\begin{array}{c}
m \\
r
\end{array}\right)_{z}=\prod_{k=1}^{r} \frac{m-r+k}{k}=\left(\begin{array}{c}
m \\
r
\end{array}\right) .
$$


The well-known formula for the sum of cosines of arguments in arithmetic progression is useful in some further computations

$$
\sum_{j=0}^{M} \cos (a+j d)=\frac{\sin \frac{(M+1) d}{2}}{\sin \frac{d}{2}} \cos \left(a+\frac{M d}{2}\right),
$$

where $M$ is a positive integer and $a, d$ are real numbers. In particular, when $d=-2 t$ and $a=M t$, the following formula is obtained

$$
\sum_{j=0}^{M} \cos (M-2 j) t=\frac{\sin (M+1) t}{\sin t} .
$$

As a consequence, we can show that the following integral formula for the binomial coefficient holds

$$
\begin{aligned}
\left(\begin{array}{c}
m \\
r
\end{array}\right) & =\frac{1}{\pi} \int_{0}^{\pi} \Lambda_{r}^{m}(t) \cdot \sum_{j=0}^{r(m-r)} \cos [r(m-r)-2 j] t \mathrm{~d} t \\
& =\frac{1}{\pi} \int_{0}^{\pi} \Lambda_{r}^{m}(t) \cdot \frac{\sin (r(m-r)+1) t}{\sin t} \mathrm{~d} t,
\end{aligned}
$$

where we have used formula (12) for $M=r(m-r)$.

\section{A formula for the coefficients of the multinomial poly- nomials}

Let $s, m, m_{1}, m_{2}, \ldots, m_{s}$ be positive integers such that $m_{1}+m_{2}+\cdots+m_{s}=m$. The multinomial polynomial is defined by the formula:

$$
\left(\begin{array}{c}
m \\
m_{1}, \ldots, m_{s}
\end{array}\right)_{z}=\frac{P_{m}(z)}{P_{m_{1}}(z) P_{m_{2}}(z) \cdots P_{m_{s}}(z)} .
$$

Clearly, for $s=2$ and $m_{1}=r$, one obtains the Gaussian polynomial. While the formula (14) seems to involve a rational function, the division actually is exact in $\mathbb{Z}[z]$.

Denoting by $M$ the degree of this polynomial, we obtain

$$
\begin{aligned}
M & =\frac{m(m+1)}{2}-\sum_{j=1}^{s} \frac{m_{j}\left(m_{j}+1\right)}{2} \\
& =\frac{1}{2}\left[m^{2}-\left(m_{1}^{2}+m_{2}^{2}+\cdots+m_{s}^{2}\right)\right] \\
& =\sum_{1 \leq k<l \leq s} m_{k} m_{l} .
\end{aligned}
$$


The factorization of the multinomial polynomial in irreducible factors involving the cyclotomic polynomials, was given by Chen and Huo in [7, Lemma 1], and naturally extends formula (3).

Proposition 4. The multinomial polynomial (14) can be factorized as

$$
\left(\begin{array}{c}
m \\
m_{1}, \ldots, m_{s}
\end{array}\right)_{z}=\prod_{k=1}^{m}\left[\Phi_{k}(z)\right]^{\lfloor m / k\rfloor-\left\lfloor m_{1} / k\right\rfloor-\left\lfloor m_{2} / k\right\rfloor-\cdots-\left\lfloor m_{s} / k\right\rfloor},
$$

where $\Phi_{k}(z)$ denotes the kth cyclotomic polynomial.

From this result we can obtain an interesting identity concerning the degree of this polynomial, which involves the floor function.

Theorem 5. Let $m, m_{1}, m_{2}, \ldots, m_{s}$ be positive integers which satisfy the identity $m=m_{1}+m_{2}+\cdots+m_{s}$. The following relation holds:

$$
\sum_{k=1}^{m} \varphi(k)\left(\lfloor m / k\rfloor-\left\lfloor m_{1} / k\right\rfloor-\left\lfloor m_{2} / k\right\rfloor-\cdots-\left\lfloor m_{s} / k\right\rfloor\right)=M .
$$

Proof. The cyclotomic polynomial $\Phi_{k}(z)$ has degree $\varphi(k)$. By Proposition 4, the left-hand side of (16) represents the degree of the multinomial in the factorization form, while the right-hand side is the degree.

In particular, from the factorization of the Gaussian polynomial (3), we obtain the following result.

Corollary 6. Let $m \geq r$ be positive integers. The following identity holds:

$$
\sum_{k=1}^{m} \varphi(k)(\lfloor m / k\rfloor-\lfloor r / k\rfloor-\lfloor(m-r) / k\rfloor)=r(m-r) .
$$

In algebraic form, the polynomial $\left(\begin{array}{c}m \\ m_{1}, \ldots, m_{s}\end{array}\right)_{z}$ can then be written as

$$
\left(\begin{array}{c}
m \\
m_{1}, \ldots, m_{s}
\end{array}\right)_{z}=\sum_{j=0}^{M} C_{j}^{m_{1}, \ldots, m_{s}} z^{j}
$$

It is known that the coefficients $C_{j}^{m_{1}, \ldots, m_{s}}, j=0, \ldots, M$, form a unimodal sequence $[1,5]$. However, as seen even in the particular case of Gaussian polynomials, this sequence is not always log-concave.

We now deduce an exact integral formula for the coefficients of the multinomial polynomials. For this purpose it is convenient to consider the function

$$
\Lambda_{m_{1}, \ldots, m_{s}}^{m}(t)=\frac{\prod_{k=1}^{m} \sin k t}{\prod_{j=1}^{s}\left(\prod_{k=1}^{m_{j}} \sin k t\right)} .
$$


Theorem 7. The coefficients of the polynomial $\left(\begin{array}{c}m \\ m_{1}, \ldots, m_{s}\end{array}\right)$ z are given by

$$
C_{j}^{m_{1}, \ldots, m_{s}}=\frac{1}{\pi} \int_{0}^{\pi} \Lambda_{m_{1}, \ldots, m_{s}}^{m}(t) \cdot \cos (M-2 j) t \mathrm{~d} t, \quad j=0, \ldots, M .
$$

Proof. By formula (6) we have

$$
P_{n}(z)=(2 i)^{n} e^{i \frac{n(n+1)}{2} t} \prod_{k=1}^{n} \sin k t
$$

hence, by using (15), the multinomial polynomial can be written as

$$
\begin{aligned}
\left(\begin{array}{c}
m \\
m_{1}, \ldots, m_{s}
\end{array}\right)_{z} & =\frac{P_{m}(z)}{P_{m_{1}}(z) \cdots P_{m_{s}}(z)}=\frac{(2 i)^{m} e^{i \frac{m(m+1)}{2} t} \prod_{k=1}^{m} \sin k t}{\prod_{j=1}^{s}(2 i)^{m_{j}} e^{i \frac{m_{j}\left(m_{j}+1\right)}{2} t} \prod_{k=1}^{m_{j}} \sin k t} \\
& =e^{i \frac{1}{2}\left[m^{2}-\left(m_{1}^{2}+\cdots+m_{s}^{2}\right)\right] t} \frac{\prod_{k=1}^{m} \sin k t}{\prod_{j=1}^{s}\left(\prod_{k=1}^{m_{j}} \sin k t\right)} \\
& =e^{i M t} \Lambda_{m_{1}, \ldots, m_{s}}^{m}(t) .
\end{aligned}
$$

Combining with the algebraic form, we obtain the following identity

$$
\left(\begin{array}{c}
m \\
m_{1}, \ldots, m_{s}
\end{array}\right)_{z}=\sum_{k=0}^{M} C_{k}^{m_{1}, \ldots, m_{s}} z^{k}=e^{i M t} \Lambda_{m_{1}, \ldots, m_{s}}^{m}(t) .
$$

It follows that

$$
C_{j}^{m_{1}, \ldots, m_{s}}+\sum_{k \neq j} C_{k}^{m_{1}, \ldots, m_{s}} z^{k-j}=z^{-j} e^{i M t} \Lambda_{m_{1}, \ldots, m_{s}}^{m}(t), \quad j=0, \ldots, M .
$$

Considering $z=\cos 2 t+i \sin 2 t$ and integrating over the interval $[0, \pi]$, we obtain the desired result.

In addition, it also results that

$$
\int_{0}^{\pi} \Lambda_{m_{1}, \ldots, m_{s}}^{m}(t) \cdot \sin (M-2 j) t \mathrm{~d} t=0, \quad j=0, \ldots, M .
$$

Notice that the integral in formula (20) is not singular, since we have

$$
\lim _{t \rightarrow 0} \Lambda_{m_{1}, \ldots, m_{s}}^{m}(t)=\frac{m !}{\prod_{j=1}^{s} m_{j} !}=\left(\begin{array}{c}
m \\
m_{1}, \ldots, m_{s}
\end{array}\right) .
$$

Similarly to the Gaussian polynomials, we have the following properties. 
Remark 8. By Theorem 7 one can easily show that the polynomial $\left(\begin{array}{c}m \\ m_{1}, \ldots, m_{s}\end{array}\right)$ z is palindromic. Indeed, for $j=0, \ldots, M$, one obtains

$$
\begin{aligned}
C_{M-j}^{m_{1}, \ldots, m_{s}} & =\frac{1}{\pi} \int_{0}^{\pi} \Lambda_{m_{1}, \ldots, m_{s}}^{m}(t) \cdot \cos (M-2(M-j)) t \mathrm{~d} t \\
& =\frac{1}{\pi} \int_{0}^{\pi} \Lambda_{m_{1}, \ldots, m_{s}}^{m}(t) \cdot \cos (-M+2 j) t \mathrm{~d} t=C_{j}^{m_{1}, \ldots, m_{s}} .
\end{aligned}
$$

One can also obtain explicit integral formulae for the middle coefficients.

Proposition 9. The middle coefficient of $\left(\begin{array}{c}m \\ m_{1}, \ldots, m_{s}\end{array}\right)$ z is given by the following formulae (depending on the parity of $M$ ):

1. If $M=2 k$, then

$$
C_{k}^{m_{1}, \ldots, m_{s}}=\frac{1}{\pi} \int_{0}^{\pi} \Lambda_{m_{1}, \ldots, m_{s}}^{m}(t) \mathrm{d} t .
$$

2. If $M=2 k+1$, then

$$
C_{k}^{m_{1}, \ldots, m_{s}}=C_{k+1}^{m_{1}, \ldots, m_{s}}=\frac{1}{\pi} \int_{0}^{\pi} \Lambda_{m_{1}, \ldots, m_{s}}^{m}(t) \cdot \cos t \mathrm{~d} t .
$$

The sum of coefficients of the multinomial polynomial $\left(\begin{array}{c}m \\ m_{1}, \ldots, m_{s}\end{array}\right)$ is

$$
\sum_{j=0}^{M} C_{j}^{m_{1}, \ldots, m_{s}}=\left(\begin{array}{c}
m \\
m_{1}, \ldots, m_{s}
\end{array}\right),
$$

obtained by taking the limit as $z \rightarrow 1$ of $\left(\begin{array}{c}m \\ m_{1}, \ldots, m_{s}\end{array}\right)$.

From Theorem 7 , we can also show that the following integral formula for the multinomial coefficients holds

$$
\begin{aligned}
\left(\begin{array}{c}
m \\
m_{1}, \ldots, m_{s}
\end{array}\right) & =\sum_{j=0}^{M} \frac{1}{\pi} \int_{0}^{\pi} \Lambda_{m_{1}, \ldots, m_{s}}^{m}(t) \cdot \cos (M-2 j) t \mathrm{~d} t \\
& =\frac{1}{\pi} \int_{0}^{\pi} \Lambda_{m_{1}, \ldots, m_{s}}^{m}(t) \cdot\left(\sum_{j=0}^{M} \cos (M-2 j) t\right) \mathrm{d} t \\
& =\frac{1}{\pi} \int_{0}^{\pi} \Lambda_{m_{1}, \ldots, m_{s}}^{m}(t) \cdot \frac{\sin (M+1) t}{\sin t} \mathrm{~d} t,
\end{aligned}
$$

where we have used the identity (12). 


\section{A formula for the coefficients of Catalan polynomials}

For a positive integer $m$, the $m$-th Catalan polynomial is defined by

$$
Q_{m}(z)=\frac{z-1}{z^{m+1}-1}\left(\begin{array}{c}
2 m \\
m
\end{array}\right)_{z}=\frac{z-1}{z^{m+1}-1} \cdot \frac{P_{2 m}(z)}{P_{m}^{2}(z)}
$$

having degree $m(m-1)$ and at least $m-1$ irreducible factors [7].

Setting $m_{1}=m_{2}=m$ in formula (22), we obtain

$$
\left(\begin{array}{c}
2 m \\
m
\end{array}\right)_{z}=e^{i m^{2} t} \frac{\prod_{k=1}^{2 m} \sin k t}{\left(\prod_{k=1}^{m} \sin k t\right)^{2}}
$$

In what follows we shall denote for simplicity

$$
\Psi^{m}(t)=\frac{\sin t}{\sin (m+1) t} \Lambda_{m}^{2 m}(t) .
$$

Theorem 10. The coefficients of the polynomial $Q_{m}(z)$ are given by

$$
c_{j}^{m}=\frac{1}{\pi} \int_{0}^{\pi} \Psi^{m}(t) \cdot \cos [m(m-1)-2 j] t \mathrm{~d} t, \quad j=0, \ldots, m(m-1) .
$$

Proof. In exponential notation, Catalan polynomials have the expression

$$
\begin{aligned}
Q_{m}(z) & =\frac{(2 i) e^{i t} \sin t}{(2 i) e^{i(m+1) t} \sin (m+1) t} e^{i m^{2} t} \frac{\prod_{k=1}^{2 m} \sin k t}{\left(\prod_{k=1}^{m} \sin k t\right)^{2}} \\
& =e^{i m(m-1) t} \frac{\sin t}{\sin (m+1) t} \Lambda_{m}^{2 m}(t) \\
& =e^{i m(m-1) t} \Psi^{m}(t) .
\end{aligned}
$$

Writing the Catalan polynomial $Q_{m}(z)$ in algebraic form it follows that

$$
Q_{m}(z)=\sum_{k=0}^{m(m-1)} c_{k}^{m} z^{k}=e^{i m(m-1) t} \Psi^{m}(t)
$$

Isolating the coefficient $c_{j}^{m}$ we deduce that

$$
c_{j}^{m}+\sum_{k \neq j} c_{k}^{m} z^{k-j}=z^{-j} e^{i m(m-1) t} \Psi^{m}(t), \quad j=0, \ldots, m(m-1) .
$$

Considering $z=\cos 2 t+i \sin 2 t$ and integrating over the interval $[0, \pi]$, the desired result follows. 
In addition, we also obtain the relations

$$
\int_{0}^{\pi} \Psi^{m}(t) \cdot \sin [m(m-1)-2 j] t \mathrm{~d} t=0, \quad j=0, \ldots, m(m-1) .
$$

Also, notice that the integral in formula (29) is not singular, since we have

$$
\lim _{t \rightarrow 0} \Psi^{m}(t)=\frac{1}{m+1}\left(\begin{array}{c}
2 m \\
m
\end{array}\right)
$$

which represents the $m$-th Catalan number.

Remark 11. By Theorem 10 one can easily show that the Catalan polynomial is palindromic. Indeed, for $j=0, \ldots, m(m-1)$, one obtains

$$
\begin{aligned}
c_{m(m-1)-j}^{m} & =\frac{1}{\pi} \int_{0}^{\pi} \Psi^{m}(t) \cdot \cos [m(m-1)-2(m(m-1)-j)] t \mathrm{~d} t \\
& =\frac{1}{\pi} \int_{0}^{\pi} \Psi^{m}(t) \cdot \cos [-m(m-1)+2 j] t \mathrm{~d} t=c_{j}^{m} .
\end{aligned}
$$

Also, we can deduce a simple integral formula for the middle coefficient.

Proposition 12. The middle coefficient of $Q_{m}(z)$ is given by:

$$
c_{\frac{m(m-1)}{2}}^{m}=\frac{1}{\pi} \int_{0}^{\pi} \Psi^{m}(t) \mathrm{d} t
$$

The sum of coefficients of $Q_{m}(z)$ is given by the formula

$$
\sum_{j=0}^{m(m-1)} c_{j}^{m}=\frac{1}{m+1}\left(\begin{array}{c}
2 m \\
m
\end{array}\right)
$$

From Theorem 10 and (31), we obtain the following integral formula for the $m$-th Catalan number:

$$
\begin{aligned}
\frac{1}{m+1}\left(\begin{array}{c}
2 m \\
m
\end{array}\right) & =\sum_{j=0}^{m} \frac{1}{\pi} \int_{0}^{\pi} \Psi^{m}(t) \cdot \cos [m(m-1)-2 j] t \mathrm{~d} t \\
& =\frac{1}{\pi} \int_{0}^{\pi} \Psi^{m}(t) \cdot \frac{\sin [m(m-1)+1] t}{\sin t} \mathrm{~d} t,
\end{aligned}
$$

where we have used the identity (12) for $M=m(m-1)$.

Acknowledgment O. Bagdasar's research was supported by a grant of the Romanian National Authority for Research and Innovation, CNCS/CCCDI UEFISCDI, project number PN-III-P2-2.1-PED-2016-1835, within PNCDI III. 


\section{References}

[1] G. Andrews, The Friedman-Joichi-Stanton monotonicity conjecture at primes, DIMACS Series in Discrete Mathematics and Theoretical Computer Science, American Mathematical Society, 2004, pp. 9-15.

[2] D. Andrica, O. Bagdasar, On cyclotomic polynomial coefficients, Malays. J. Math. Sci., in: Proceedings of "Groups, Group Rings, and Related Topics - 2017" (GGRRT 2017), 19 - 22 Nov 2017, Khorfakan, UAE (2018) (to appear).

[3] D. Andrica, O. Bagdasar, Some remarks on a general family of complex polynomials, (2018) (submitted).

[4] D. Andrica, O. Bagdasar, On some results concerning the polygonal polynomials, Carpathian J. Math. (2019) (to appear).

[5] S. Brunetti, A. D. Lungo, On the polynomial $\frac{1}{[n]_{q}}\left(\begin{array}{l}n \\ k\end{array}\right)_{q}$, Adv. Appl. Math. 33 (2004), no. 3, 487-491.

[6] L. M. Butler, The q-log-concavity of q-binomial coefficients, J. Comb. Theory A 54 (1990), no. 1, 54-63.

[7] W. Y-C. Chen, Q-H. Hou, Factors of the Gaussian coefficients, Discrete Math. 306 (2006), no. 13, 1446-1449.

[8] A. N. Kirillov, Unimodality of generalized Gaussian coefficients, arXiv preprint hep-th/9212152 (1992).

[9] D. E. Knuth, H. S. Wilf, The power of a prime that divides a generalized binomial coefficient, J. Reine Angew. Math. 396 (1989), 212-219.

[10] K. M. O'Hara, Unimodality of Gaussian coefficients: a constructive proof, J. Comb. Theory A 53 (1990), no. 1, 29-52.

[11] R. P. Stanley, Log-Concave and Unimodal Sequences in Algebra, Combinatorics, and Geometry, Ann. NY Acad. Sci. 576 (1989), no. 1 Graph Theory, 500-535.

[12] B. D. Sullivan, On a conjecture of Andrica and Tomescu, J. Integer Seq. 16 (2013), Article 13.3.1.

Dorin ANDRICA

Faculty of Mathematics and Computer Science,

Babeş-Bolyai University of Cluj-Napoca,

Kogălniceanu Street, Nr. 1, 400084 Cluj-Napoca, Romania.

Email: dandrica@math.ubbcluj.ro

Ovidiu BAGDASAR

Department of Electronics, Computing and Mathematics

University of Derby

Kedleston Road, Derby DE22 1GB, England, UK.

Email: o.bagdasar@derby.ac.uk 